This is an author produced version of a paper published in Osteoporos Int. This paper has been peer-reviewed but does not include the final publisher proof-corrections or journal pagination.

Citation for the published paper:

Holmberg, A and Johnell, $\mathrm{O}$ and Nilsson, $\mathrm{P}$ and Nilsson, J and Berglund, $\mathrm{G}$ and Akesson, $\mathrm{K}$

"Risk factors for fragility fracture in middle age. A prospective population-based study of 33,000 men and women."

Osteoporos Int. 2006, May 4.

10.1007/s00198-006-0137-7

Access to the published version may require journal subscription.

Published with permission from: Springer 


\section{Risk Factors for Fragility Fracture in Middle Age. A prospective population-based study of 33000 men and women.}

Anna H Holmberg ${ }^{1}$, Olof Johnell ${ }^{1}$, Peter M Nilsson ${ }^{2}$, Jan-Åke Nilsson ${ }^{1}$, Göran Berglund ${ }^{2}$ and Kristina Åkesson ${ }^{1}$.

Clinical and Molecular Research Unit ${ }^{1)}$ and Internal Medicine Research Unit ${ }^{2)}$, Department of Clinical Sciences Malmö, Lund University, Malmö University Hospital, SE20502 Malmö, Sweden.

\section{Correspondence to:}

Kristina Åkesson Dept. of Orthopaedics, Malmö University Hospital, 20502 Malmö, Sweden.

Phone: +46-40-336123

Fax: +46 40-33 6200

E-Mail: kristina.akesson@med.lu.se 


\section{Abstract}

Background: The incidence of fragility fractures begins to increase in middle age. We investigated prospectively risk factors for low-energy fractures in men and women, and specifically for forearm, proximal humerus, vertebral and ankle fractures.

Methods: The population-based Malmö Preventive Project consists of 22444 men and 10902 women, mean age 44 and 50 years at inclusion. Baseline assessment included multiple examinations and lifestyle information. Mean follow-up: 16 and 11 years for men and women, respectively, regarding incident fractures. Fractures were ascertained from radiographic files.

Results: At least one low-energy fracture occurred in 1262 men and 1257 women. In men, the risk factors most strongly associated with low-energy fractures were diabetes (RR 2.38, CI 95\% 1.65-3.42) and hospitalization for mental health problems (RR 1.92, CI 95\% 1.47-2.51). Factors associated with mental health and lifestyle significantly increased the fracture risk in most of the specific fracture groups; hospitalizations for mental health problems (RR 2.28 to 3.38), poor appetite (RR 3.05 to 3.43), sleep disturbances (RR 1.72 to 2.95), poor, self-rated health (RR 1.80 to 1.83 ) and smoking (RR 1.70 to 2.72 ).

In women, the risk factors most strongly associated with low-energy fractures were diabetes (RR 1.87, CI $95 \%$ 1.26-2.79) and previous fracture (RR 2.00, CI $95 \%$ 1.56-2.58). High body mass index significantly increased the risk of proximal humerus and ankle fractures (RR 1.21 to 1.33 ), while, by contrast lowering the risk of forearm fractures (RR 0.88 , CI $95 \% 0.81$ 0.96 ).

Conclusion: Risk factors for fracture in middle-aged men and women are similar, but with gender differences, for forearm, vertebral, proximal humerus and hip fracture, whereas risk factors for ankle fractures differ to a certain extent. The risk factor pattern indicates a generally impaired health status with mental health problems as a major contributor to fracture risk, particularly in men.

Key words: Fragility fractures, risk factors, diabetes, mental health 


\section{Introduction}

Over recent decades the incidence of fractures has increased substantially, especially among the elderly $[1,2]$. Different fracture types dominate at different ages and the fracture pattern varies between women and men [1]. In middle age, forearm and ankle fractures are the most frequent fractures, whereas in the elderly hip fractures become the predominating problem. Fracture incidence is usually greater among men than women before the age of 50 years, but at higher ages women suffer more fractures [3]. In general, 75 percent of all fractures among men occur before the age of 45 , whereas in women 75 percent of all fractures occur after the age of 45 [4].

Although the consequences of a limb fracture are usually not as devastating as those of a hip fracture, the suffering, inconvenience and the temporary or sometimes permanent disability of the individual are strong enough reasons to motivate a closer epidemiological investigation of these fractures. The fracture-related costs to the society are substantial; the cost for the direct medical care of osteoporotic fractures other than hip fractures in the USA was 5.1 billion dollars in 1995 [5]. Prevention of these fractures would free resources for other areas of health care and avoid unnecessary suffering.

Having a history of a previous fracture is a known risk factor predisposing for future fractures in the elderly [6-8]. The identification of factors associated with fracture risk already in middle age, when a fracture is still a rare event, has not been extensively investigated. Increased knowledge in this area would ultimately enable us to initiate preventive measures when most appropriate.

The primary objective of this study was to investigate the risk factor pattern for fractures commonly occurring in women and men in middle age, 48-68 years. A secondary objective was to identify differences in risk between men and women. 


\section{Material and methods}

The Malmö Preventive Project is a prospective, population-based, cardiovascular screening study consisting of 33346 probands, 22444 men and 10902 women, representing $72 \%$ of the invited population [9]. Mean age at the baseline investigation was 44 (range 27-61) years for men and 50(range 28-58) years for women. The inclusion period for men was 1974 to 1984 (10 years) and for women from 1977 to 1992 (15 years). The primary objective of the Malmö Preventive Project was to describe cardiovascular risk factors and related conditions in a middle-aged population and to improve the scientific basis for cardiovascular disease prevention. Over and above this, the study provides data for evaluation of other common public health conditions such as fracture and diabetes. The probands were followed prospectively till the end of 1999, with a mean follow-up of 19 years (range 7-25 years) for men and 15 years (7-22 years) for women for incident fractures and mortality.

\section{Physical examination}

The baseline physical investigation included height and body weight measurement as well as triceps skinfold thickness. Blood pressure and pulse rate were measured twice after a 10minute rest and the mean was recorded. Simple spirometry (Spirotron ${ }^{\circledR}$, Drägerwerke, Germany) was performed and pulmonary function parameters were calculated. In a subgroup of the women, bone mineral density of the distal radius was measured and has been reported previously [10].

\section{Questionnaire}

At baseline the participants in the study completed a questionnaire regarding health-related and lifestyle issues, previously described in detail [11]. During the extended inclusion period certain questions were added while others were withdrawn. The total number of questions used in the questionnaire were 417,78 of which were core questions asked throughout the 
inclusion period. The mean response rate was for the women about $98 \%$ and for the men 72 $\%$ for the core questions. In the early period of this study (the beginning of the seventies) the computer technique was still young, and due to computer problems some of the questionnaire results were lost for 6368 men. The response rate was $99 \%$ for the remaining results. Questions that were added during the inclusion period had a response rate of almost $100 \%$ for those who had the opportunity to respond.

The amount of data is extensive, thus only variables of biological interest and with possible impact on fracture risk were selected for this report.

\section{Laboratory investigation}

Morning blood samples were collected from the participants after an over-night fast. The following analyses were performed and used in this substudy; haematoglobin, erythrocyte sedimentation rate (ESR), serum creatinine, fasting blood glucose, serum $\gamma$-glutamyl transferase, serum triglycerides, serum total cholesterol, serum uric acid and serum phosphate. Due to skewed distribution of serum $\gamma$-glutamyl transferase, data were logarithmically transformed when used in the analyses.

\section{Fracture identification}

The fracture data were obtained by linking the probands included in the Malmö Preventive Project with the register at the Department of Diagnostic Radiology at Malmö University Hospital. In the city of Malmö all emergency radiographic examinations are performed at the Department of Diagnostic Radiology at Malmö University Hospital, the fractures are recorded and the films are stored and saved permanently. The unique 10-digit personal identification number, based on birth date and issued to every Swedish citizen, makes identification of cases easy and precise. The fractures identified were confirmed through manual search of the medical and radiological files. Previous studies have shown that at least $97 \%$ of all fractures 
experienced by citizens of Malmö can be identified this way [12]. The fractures registered were classified under the following categories, namely, forearm, vertebral, proximal humerus and ankle fractures (Table 1). Data on hip fractures, previously reported [11], are included for comparison. Regarding vertebral fractures, only those coming to clinical attention and described in radiographic reports, or accidentally found on chest or abdominal x-rays for other causes and described in the radiographic report were included. The degree of deformity was not quantified.

The fractures were classified as high or low-energy trauma depending on the cause of fracture. Fractures caused by falling from standing height or less were classified as lowenergy fractures and those caused by high-energy trauma as high-energy fractures. The classification was based on information given in the radiographic reports. Of all fractures, $4098(97.7 \%)$ were described with adequate information about the degree of trauma in the radiology reports. The 98 fractures with insufficient information concerning trauma were classified as low-energy fractures, based on the experience that it is highly unlikely that information about high-energy accidents is not reported.

Fractures caused by high-energy trauma were excluded from the analyses, as were pathological fractures caused by cancer or other bone diseases. 


\section{Statistics}

For this substudy, relevant data were extracted from the Malmö Preventive Project main database. The population was divided into those with and those without fracture. Individuals suffering exclusively fractures caused by high-energy were excluded from the fracture population and included in the non-fracture population. Individuals with at least one lowenergy incident fracture were classified into the low-energy fracture group, even if a previous or subsequent high-energy fracture was recorded during the study period. The first fracture of each fracture type was the one included in the calculation, even if the individual had suffered more than one fracture of the same type. If an individual suffered more than one type of fracture, the individual was included in the calculations for each fracture type, albeit under different dates for the fracture incident. Fracture incidence was calculated as the number of fractures per 1000 person years.

Baseline descriptive data of the whole cohort and the fracture subgroups were presented as percentages, means and standard deviations (SD). Due to the skewed distribution of serum $\gamma-$ glutamyl transferase it was logarithmically transformed when used in the analyses. Variables of biological interest were chosen from the database. Z-scores were calculated for the continuous variables, to permit comparison of different variables. The variables were analyzed, one by one, in an age-adjusted Cox proportional hazard model. To reduce the risk of mass significance through multiple testing we chose a p-value of $<0.01$ as the level of significance. A final multiple regression model was constructed consisting of 11 (women) and 10 (men) variables. The variables were selected from the age-adjusted Cox proportional hazard model based on the strength of their association with the different fracture types. Among variables with possible interdependence we chose the one with the strongest association and highest response rate. Two questions; one regarding previous fracture history 
and another regarding hormone replacement therapy, were not included throughout the study, thus generating an incomplete data set. Despite their significant impact on fracture risk we chose to exclude these questions from the final Cox analysis.

The statistics program used was SPSS 12.0 for Windows (Statistics package for Social Sciences, Chicago, Illinois, USA). 


\section{Results}

\section{Descriptives}

\section{Women}

During the 15-year follow-up, 1878 incident fractures were registered affecting 1292 women, i.e. $12 \%$ of the female study population. Of these, 1257 women $(97 \%)$ were classified as suffering low-energy fractures. The number of women with only one low-energy fracture was 894, representing $71 \%$ of the women with low-energy fractures. Of the total number of 1805 low-energy fractures recorded, 662 were forearm fractures, 155 proximal humerus, 160 vertebral, 223 ankle, and 141 hip fractures (Table 1). Fracture incidence rates for each type of fracture are reported in figure 1 . The age effect is most pronounced in women.

Baseline characteristics are reported for the entire cohort and for the major fracture types (Table 2). The women suffering fractures were slightly older at study start than the nonfracture population, with the exception of women with ankle fractures. Mean body weight was $3.5 \mathrm{~kg}$ greater at inclusion in women with ankle and proximal humerus fractures compared to the non-fracture population, and $2.5 \mathrm{~kg}$ less in women with a hip fracture. The prevalence of diabetes seemed higher in women with ankle, vertebral and hip fractures. Women with vertebral and hip fractures also appeared to suffer from more comorbidities than the nonfracture population.

\section{Men}

During the 19-year follow-up 2422 incident fractures were registered affecting 1505 men, i.e. $6.7 \%$ of the male study population. Of these, 1262 men (84\%) were classified as suffering low-energy fractures. The number of men with only one low-energy fracture was 822 , representing $65 \%$ of the men with low-energy fractures. Of the total number of 1975 lowenergy fractures recorded, 330 were forearm fractures, 123 proximal humerus, 168 vertebral, 
259 ankle, and 174 hip fractures (Table 1). The fracture incidence rates were half of those in women and the age effect was seen only in the highest age group (Fig1).

Men with fractures were slightly older at the start of the study than the non-fracture population, which was especially apparent in men with a hip fracture (Table 2). Mean body weight and BMI were slightly lower in all separate fracture groups except for ankle fractures, where it was slightly higher. Smoking was more common among men suffering from vertebral, proximal humerus and hip fractures.

Risk factors for low-energy fractures overall and for specified types of fracture, for women and for men, are reported separately. The factors evaluated are presented as means and standard deviations in Table $3 \mathrm{a}$ and $\mathrm{b}$. In the following we will highlight the results for women and men in the uni- and multivariate models presented in Table 4 -7.

\section{Risk factors in women}

\section{Low energy fractures}

The risk factor with the largest impact on low energy fractures in general was a history of previous fracture, doubling the fracture risk (RR 2.00, CI $95 \% 1.56-2.58, \mathrm{p}=0.001)$ Diabetes was associated with a similar risk increase $(R R 1.95,1.33-2.86, p=0.001)$, while high serum $\gamma$ -glutamyl transferase and poor, self-rated health gave smaller risk increases. A relative risk decrease was seen for those using Hormone Replacement Therapy (HRT). In the final Cox model, diabetes remained a significant risk factor of low energy fractures. (Tables 4 and 6 )

\section{Forearm fractures}

The risk factor with the largest impact on forearm fractures was a history of previous fracture, doubling the risk ( $R R 2.00,1.40-2.85, \mathrm{p}=0.001)$, whereas diabetes was not associated with an 
increased risk of forearm fracture. High BMI was associated with decreased risk of forearm fracture.

\section{Vertebral fractures}

Diabetes was the risk factor with the largest impact on vertebral fractures, increasing the fracture risk more than three times $(\mathrm{RR} 3.56,1.75-7.23, \mathrm{p}=0.001)$. A history of previous fracture $(\mathrm{RR} 3.13,1.67-5.85, \mathrm{p}=0.001)$ also increased the fracture risk, as did high serum $\gamma$ glutamyl transferase, smoking and decreased lung capacity (FVC) but to a lesser extent. ESR as an indicator of inflammation was also associated with a small risk increase (RR 1.16, 1.04$129, \mathrm{p}=0.009)$.

\section{Proximal humerus fractures}

In contrast to most of the other fracture groups the risk of proximal humerus fracture was not affected by previous fracture history, diabetes, smoking or high serum $\gamma$-glutamyl transferase. The factor most strongly associated with proximal humerus fracture was being in the higher age segment of the study population, with RR $2.27(1.76-2.93, \mathrm{p}=0.001)$. High body weight, high BMI and poor, self-rated health also increased the fracture risk.

\section{Ankle fractures}

A history of previous fracture doubled the risk of ankle fracture (RR 2.11, 1.17-3.80, $\mathrm{p}=0.001$ ), whereas the variable most strongly associated with ankle fractures was diabetes (RR 3.36, 1.58-7.15, $\mathrm{p}=0.002$ ). High serum $\gamma$-glutamyl transferase as well as high body weight and BMI increased the risk of ankle fracture. 


\section{Risk factors in men}

\section{Low energy fractures}

The factor associated with the strongest risk effect on low energy fractures in general was suffering from diabetes, which more than doubled the fracture risk (RR 2.50, 1.75-3.57, $\mathrm{p}=0.001)$. A history of hospitalization for mental disorders $(\mathrm{RR} 1.92,1.47-2.51, \mathrm{p}=0.001)$ and having a poor appetite (RR 1.72, 127-2.32, $\mathrm{p}=0.001)$ also increased the low energy fracture risk. Smoking and a high serum $\gamma$-glutamyl transferase, an indirect measure of an alcohol effect, were associated with moderately increased risks of fracture. High BMI was associated with a decreased risk of low energy fractures in men, it is a protective attribute. In the final Cox model the associations remained chiefly the same. (Tables 5 and 7)

\section{Forearm fractures}

The factor associated with the strongest risk effect on forearm fractures was hospitalization for mental disorders (RR 2.28, 1.37-3.78, $\mathrm{p}=0.001)$. The only other factor significantly associated with forearm fractures in men was high BMI (RR 0.85, 0.75-0.96, p=0.007), which decreased the risk of fracture.

\section{Vertebral fractures}

A history of hospitalization for mental disorders increased the fracture risk significantly (RR 2.34, 1.36-4.03, $\mathrm{p}=0.002)$. Other strong risk factors for men with vertebral fractures were having a poor appetite (RR 3.05, 1.80-5.17, $\mathrm{p}=0.001)$ and being on sick leave at the time of the baseline investigation (RR 2.98, 1.83-4.85, $\mathrm{p}=0.001)$. Smoking and a high serum $\gamma$ glutamyl transferase were also associated with increased risks of vertebral fracture. 


\section{Proximal humerus fractures}

For proximal humerus fractures several factors related to mental health were significant contributors to fracture risk, each increasing the fracture risk three-fold; prior hospitalization for mental disorders, poor appetite and premature awakening. Smoking and high serum $\gamma-$ glutamyl transferase were also associated with increased risks of proximal humerus fracture. In men a high BMI decreased the risk of proximal humerus fracture.

In the final Cox model a high level of serum triglycerides was associated with a decreased risk of proximal humerus fractures.

\section{Ankle fractures}

The significant risk factors for ankle fractures were to some extent similar to other peripheral fractures in men but with a lower impact; prior hospitalization for mental disorders (RR 2.74, 1.62-4.66, $\mathrm{p}=0.002$ ), premature awakening and high serum $\gamma$-glutamyl transferase.

In the final Cox model only the association between high serum $\gamma$-glutamyl transferase and ankle fracture remained. 


\section{Discussion}

The aetiology of fractures is multi-facetted and complex, making fracture studies and reporting difficult and never complete. Our aim has been to determine and examine a range of factors that affect fracture risk in middle age, low energy fractures in general, and in more detail fractures of the forearm, vertebrae, proximal humerus and ankle. Low energy fractures in general were, in women, strongly associated with diabetes and previous fracture history, and in men with diabetes and mental health problems. For the specified fracture groups, the risk factor pattern was to some extent different, but certain factors affected almost all the subgroups; in women being in the higher age segment of the study population, a history of previous fracture and diabetes. In men being in the higher age segment of the study population, low BMI, high serum $\gamma$-glutamyl transferase, smoking, sleep disturbances and hospitalization for mental disorders were all factors identified as significant contributors to risk in most of the fracture subgroups. Our findings suggest that individuals in middle age with impaired physical and mental health are at high risk of experiencing peripheral and vertebral fractures.

Fracture incidences were calculated for the specified fracture groups. The results are similar to those of a study of the Geelong population in Australia, except for forearm fractures [13]. The discrepancies may be attributable to the low number of fractures in some categories. Our incidence curves are similar to the results of a register study by Kanis and co-workers performed on the total population of Malmö through fracture registers, although our incidence rates are lower [14]. However, our incidence rates are similar to earlier clinical studies in Malmö [1] but as previously recognised, the rates for some of the fractures, forearm fractures in particular, are higher than in other, large epidemiological studies [15]. It cannot be excluded that this study, as well as other population-based cohort studies, attract a somewhat 
more health-conscious segment of the population, who thus sustain fewer fractures, in contrast to a register study that includes all citizens.

In both men and women, high body weight and BMI lowered the risk of forearm and hip fractures, in men also of proximal humerus fractures. In contrast, in women high body weight and BMI increased the risk of proximal humerus and ankle fractures. This further elicits the complexity of fracture aetiology, when both being overweight and being thin affects fracture risk, albeit different types of fracture. One could speculate that there is an association between poor nutrition and poor bone quality, as well as between high body weight and clumsiness, with an increased tendency to fall and a higher impact of a fall even from standing height. These findings suggest two different types of individuals, both prone to fracture but with different risk factors predominating. A case-control study of 448 men and women over 45years of age found that high BMI as well as frequent falling were risk factors for proximal humerus fracture [16]. This is consistent with our findings in women. Proximal humerus fractures, at least in elderly subjects, have been associated with low bone mineral density [17] and considered a type fracture of osteoporosis. A study by Olsson and co-workers of both middle-aged and elderly subjects showed associations between humerus fractures and previous fracture history as well as increased risk of future fracture [18]. This further supports our hypothesis about subgroups within the fracture population.

High body weight and BMI increased the risk of ankle fractures in women but decreased the risk of forearm and hip fractures. In the age-adjusted Cox model we found other risk factors common to these fracture types, but in the final Cox model only BMI remained a significant risk factor for ankle fractures and a factor decreasing risk of hip fractures. The age effect was no longer significant for ankle fractures, in contrast to the other fracture types. This is 
consistent with previous reports [10,19-22] suggesting that risk factors for ankle fractures are related to lifestyle including a physically active life, whereas the risk associated with forearm fractures is more dependent on bone density. Our results indicate that ankle fractures differ in risk factor profile compared to fractures commonly associated with poor bone quality, and it is thus questionable to include these in an analysis of osteoporotic fractures, a point which is further supported by a report by Kanis and co-workers [4].

Diabetes, in both men and women, was strongly associated with an increased relative risk of low energy fractures and hip fractures, in women also of vertebral and ankle fractures, a result consistent with previous studies [23-27]. In the multivariate analysis the association between ankle fractures and diabetes in women disappeared. This is probably due to the small number of cases of diabetes found in each specific fracture group, and not to a lack of impact from diabetes on ankle fractures. Where diabetes is of long duration, multiple organ systems of the body are affected, leading to angiopathy, neuropathy and nephropathy, with effects on bone mass and on the tendency to fall. In a previous study [11] we have shown that diabetes has a large impact on hip fracture risk already in middle-age, suggesting more fragile bone structure in individuals suffering from diabetes.

Blood lipids have in recent studies been associated with BMD, reporting a positive correlation between serum-triglycerides and BMD and a negative correlation between serum-cholesterol and BMD $[28,29]$. In this study we have found that in both men and women of middle age, high levels of serum-triglycerides are associated with a decreased risk of some peripheral fracture types. A high level of blood lipids may possibly represent a good status concerning nutrition and a good health, protecting the individual from fracture. The true correlation between blood lipids and fracture risk remains to be explored. 
Elevated levels of serum $\gamma$-glutamyl transferase may be an indicator of alcohol overconsumption [30-32]. Also in the population studied alcohol over-consumption has been shown to be the main cause of elevated levels of $\gamma$-glutamyl transferase [33]. However, indicators of the metabolic syndrome, including liver derived serum lipids, may influence the levels of $\gamma$-glutamyl transferase, and the likelihood of interaction is high. Nevertheless alcohol was still a major contributor [34]. In this study elevated serum $\gamma$-glutamyl transferase was associated with an increased risk of low energy fractures in general in both men and women for most of the specified fracture subtypes. Studies have shown that alcohol overconsumption increases the risk of fracture through metabolic effects as well as alcohol-related falls and a more hazardous life style in general [35-37]. The fracture pattern of the men in this study, with a high proportion of high-energy fractures as well as hand and skull fractures, may indicate an unsafe life style, possibly in connection with alcohol abuse. In women elevated serum $\gamma$-glutamyl transferase levels were associated with an increased risk of vertebral and ankle fractures. One could speculate that the effect on ankle fractures may be a result of acute alcohol intoxication, since ankle fractures seem to be more associated with lifestyle factors than poor bone quality, and the effect on vertebral fractures seem more related to long-term effects of alcohol consumption. Unfortunately the study did not include specific questions on alcohol consumption, thus we rely on this indirect but nevertheless objective measure.

In many previous reports smoking has been found to be a risk factor for fractures in general and hip fractures in particular [38-40] with a negative effect on $\operatorname{BMD}[41,42]$. In this study smoking was associated with increased risk of vertebral fractures in women, and in men with low energy fractures in general and with vertebral, proximal humerus and hip fractures. 
Several variables regarding psychological well-being are recorded, allowing us to evaluate the impact on fracture risk. In men one variable was a significant risk factor for all types of fracture, namely previous hospitalization for mental health problems. When we compared the individuals reporting mental health problems with the non-fracture population, these individuals appeared to suffer from more comorbidities than the non-fracture population. A study from Tromsö, Norway reported psychiatric disease in men as a risk factor for all fracture types and especially for forearm and proximal humerus fractures, in agreement with our findings [43]. Individuals with mental health problems may lead a more irregular life, with possible alcohol and drug abuse affecting their health, creating a frailer body constitution and also with an increased risk of falling. Similar reasoning may be applicable regarding the increased risk of vertebral fractures in those currently on sick leave, assuming that sick leave is a sign of co-morbidity.

This study has some limitations. Since the original study was not designed to investigate fractures and fracture risk and because of computer error, certain data sets are incomplete and information is accessible only for the major parts of the study population. Nevertheless, the overall response rate is excellent. BMD measurements were performed only on a small part of the population [10], thus the level of prevalent osteoporosis cannot be determined.

A strength of this study is that, to our knowledge, this is one of the first prospective studies evaluating a broader range of risk factors of common fracture types in middle age in both men and women. Furthermore, we have been able to evaluate factors that are not commonly studied, which should be regarded as a strength. The study is large and with a long follow-up, which makes the results more reliable. 
This study has identified and described many risk factors for low-energy fractures in middle age, and their impact on different fracture types. This confirms the multitude and complexity of factors affecting fracture risk. The study indicates similarities in the risk factor patterns between forearm, vertebral, proximal humerus and hip fractures, whereas the study indicates that ankle fractures to a certain extent have a different risk profile. A middle-aged person of generally impaired physical and mental health status with subsequent impaired bone strength as a possible background factor is, according to this study, at high risk of a peripheral fracture. Identification of risk factors in younger age groups gives us a unique opportunity to implement preventive strategies at a time when intervention may still prevent irreversible bone loss.

\section{Acknowledgements}

The Swedish Research Council Project K2003-73X-11610-08A, The Kock Foundation, The Herman Järnhardt Foundation, the Malmö University Hospital Funds and regional research grants, supported this study. 


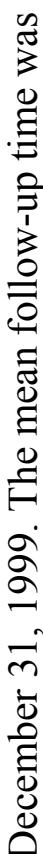

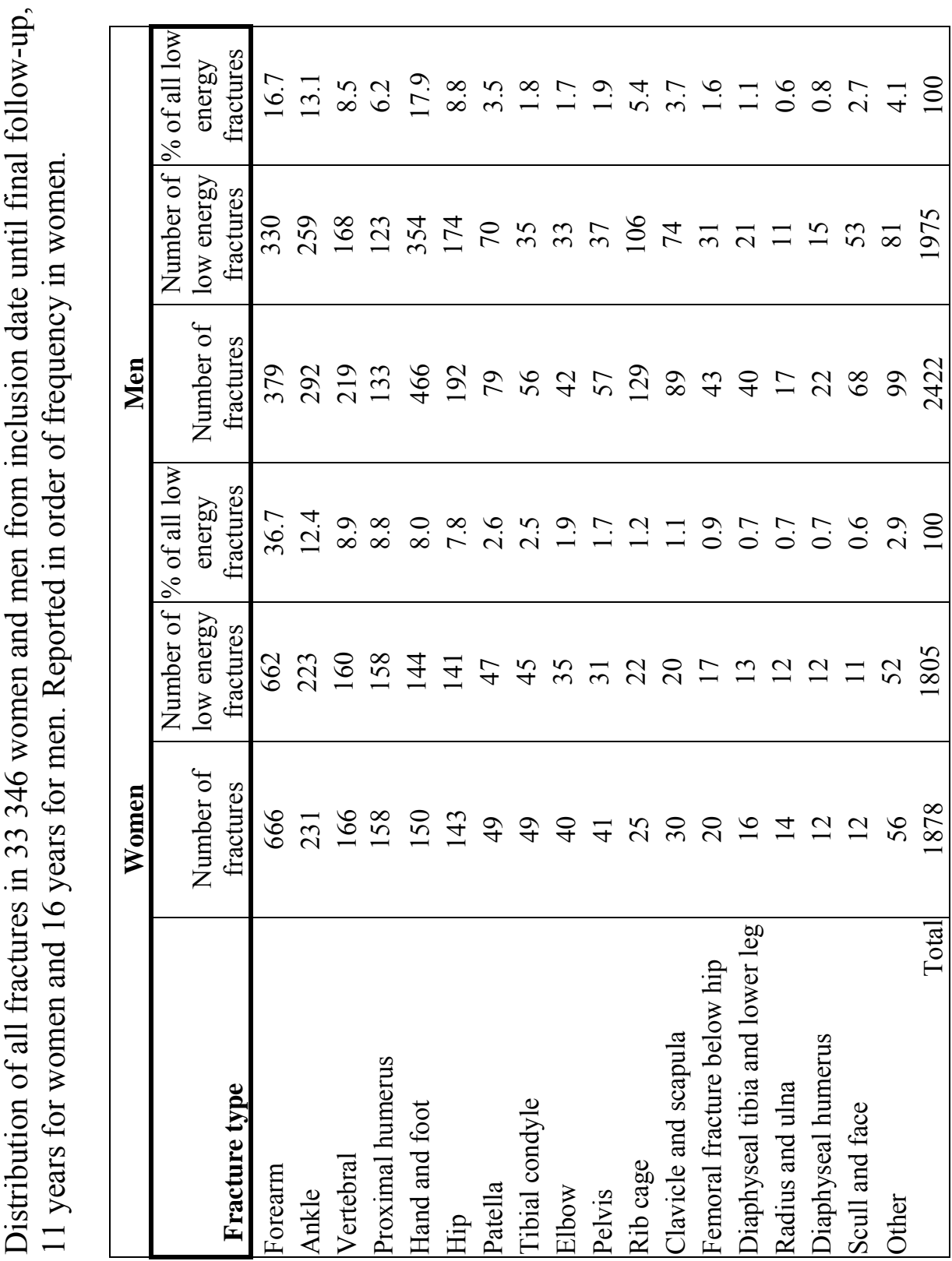




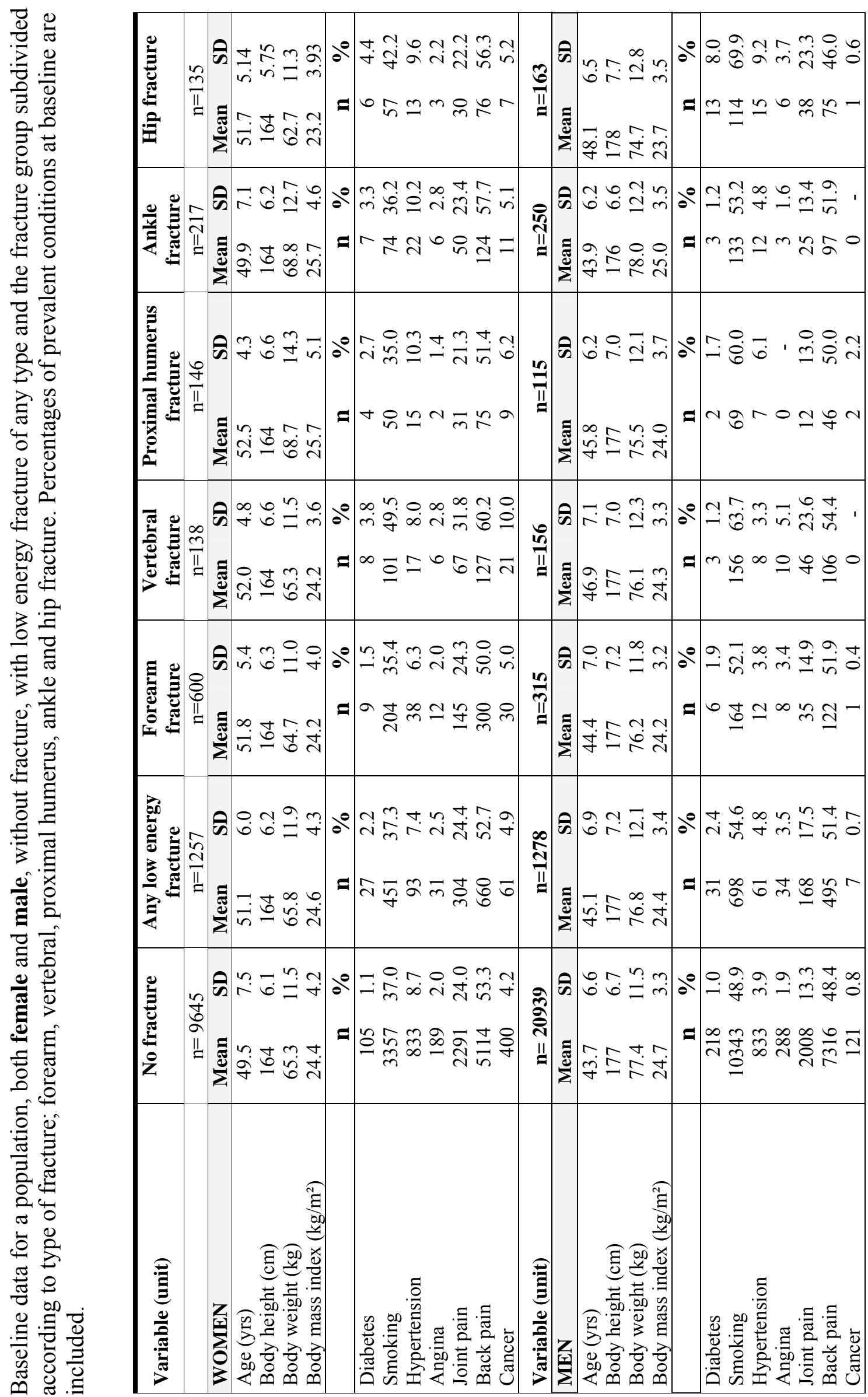




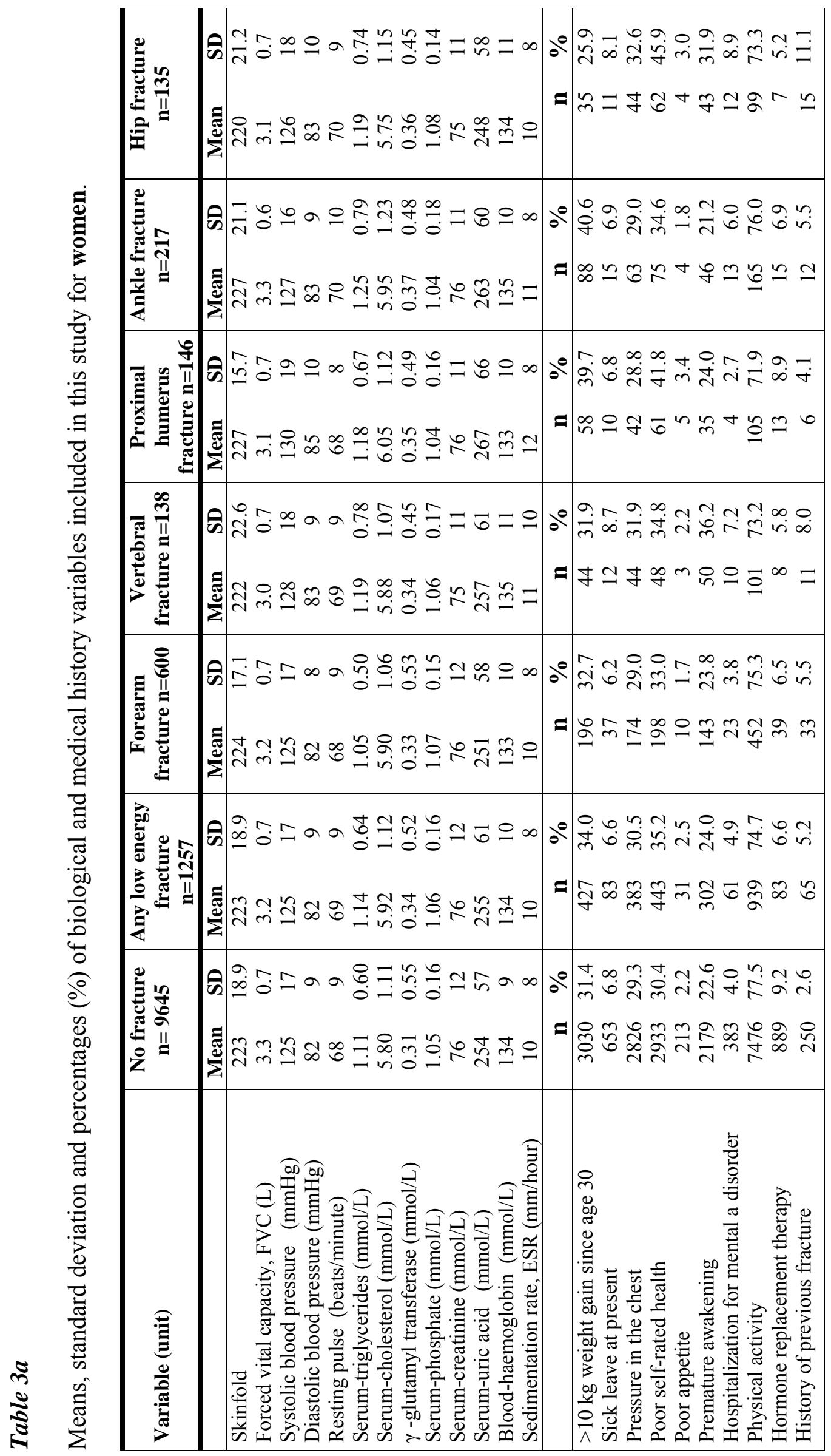




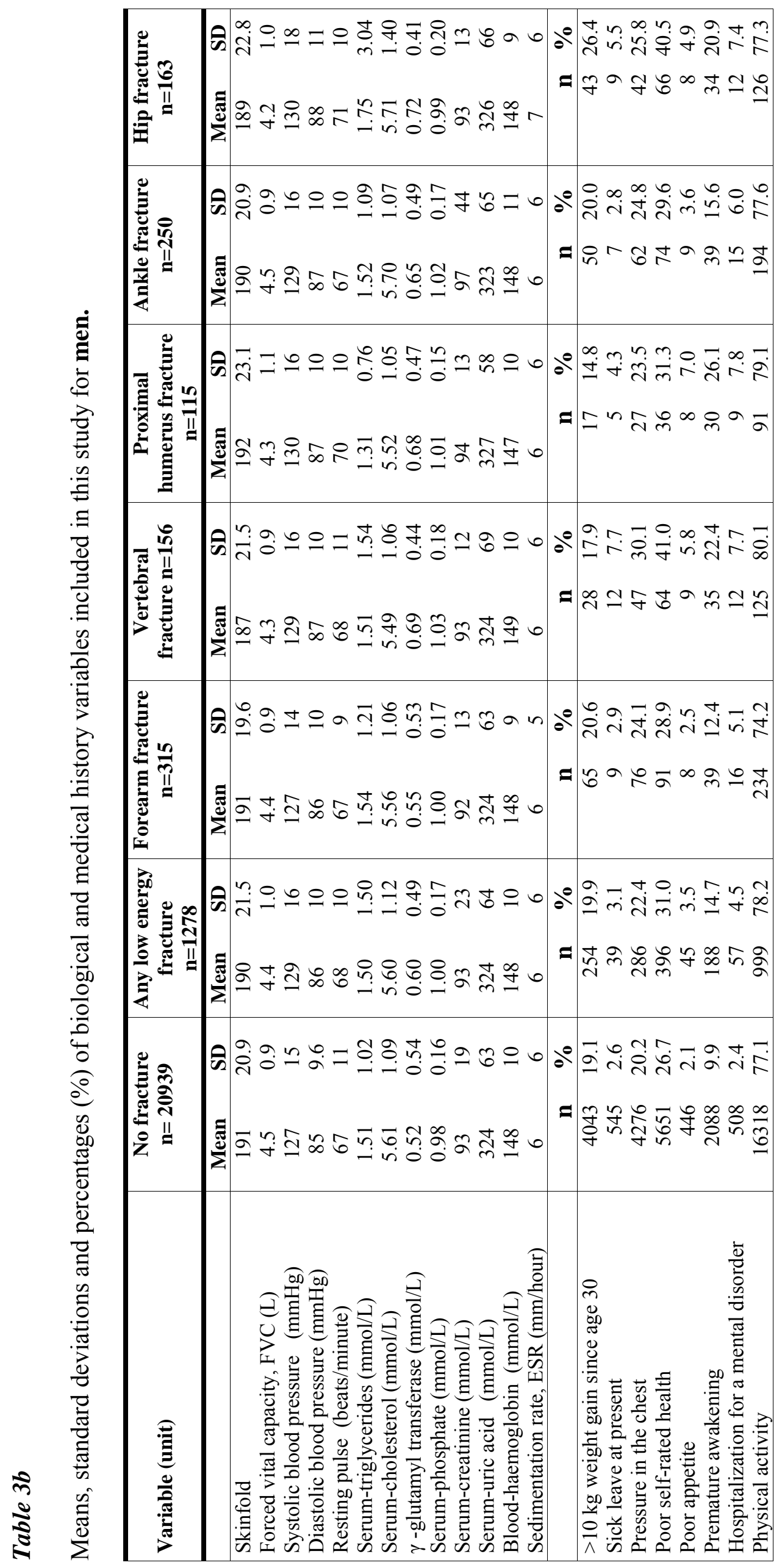




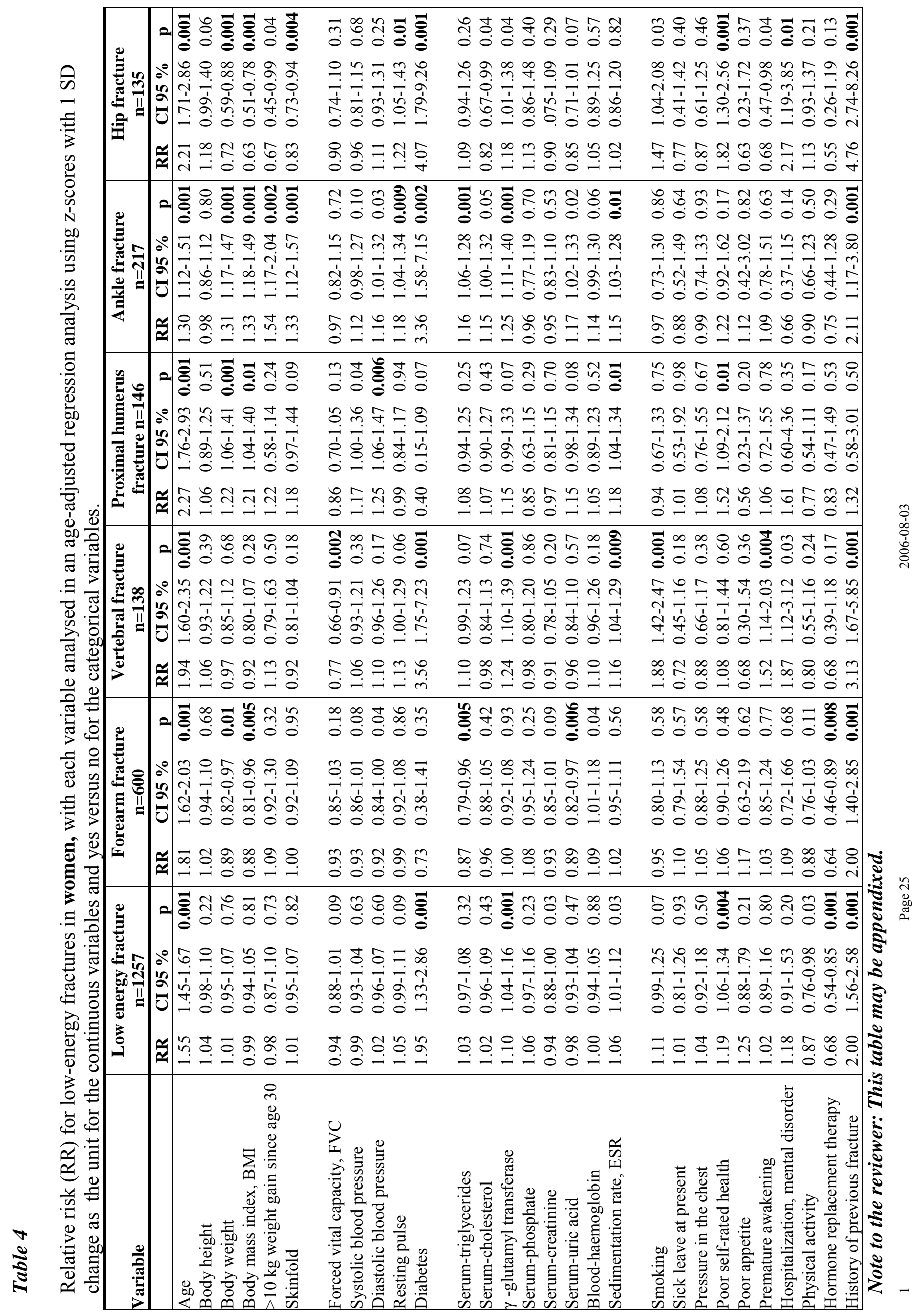




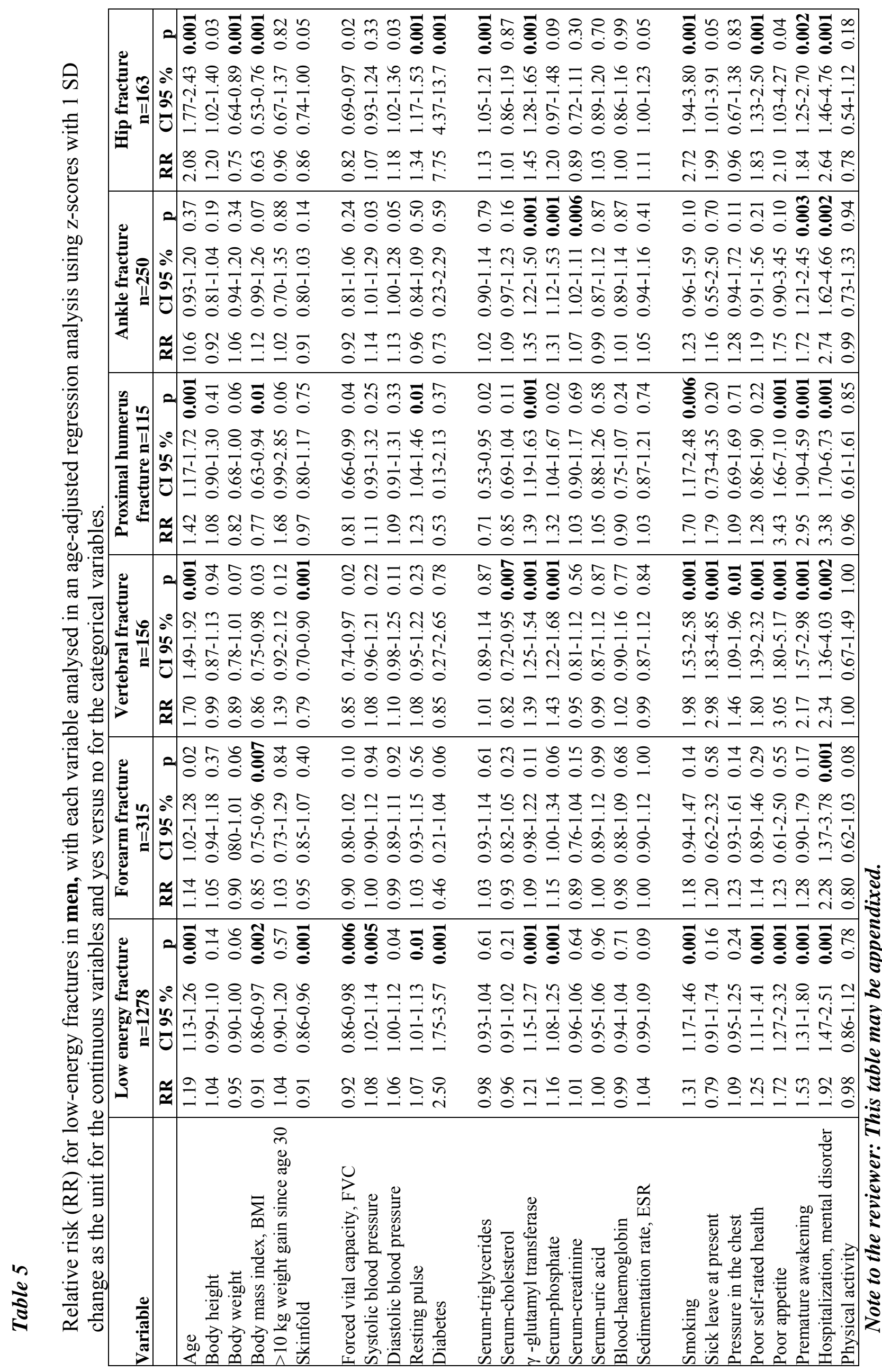




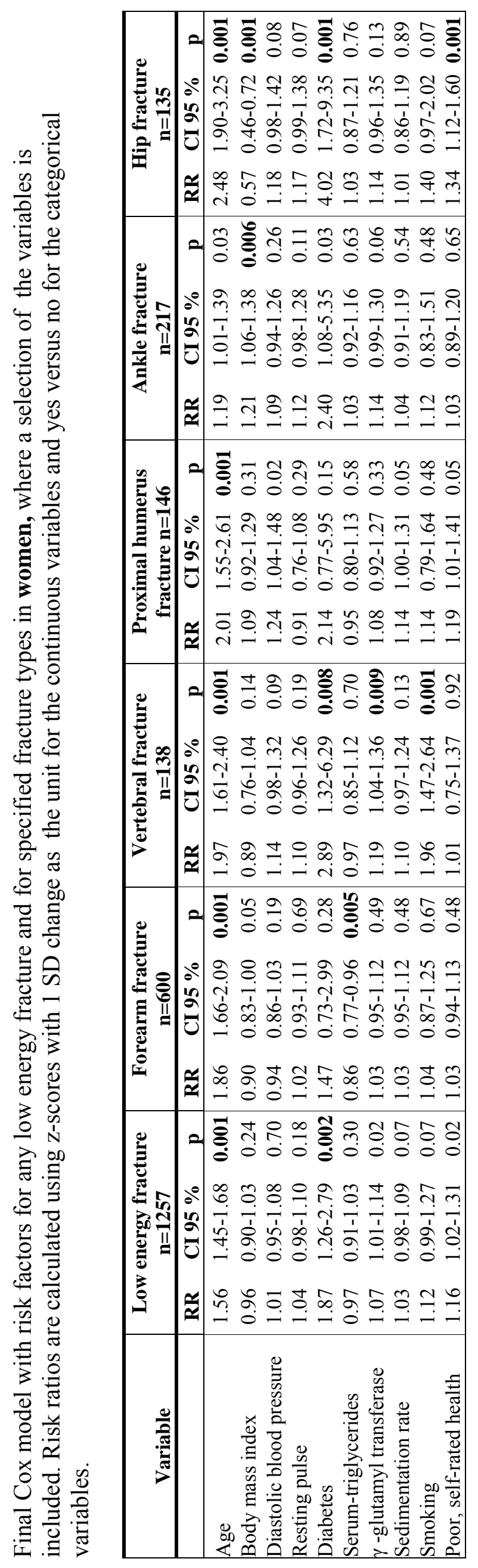




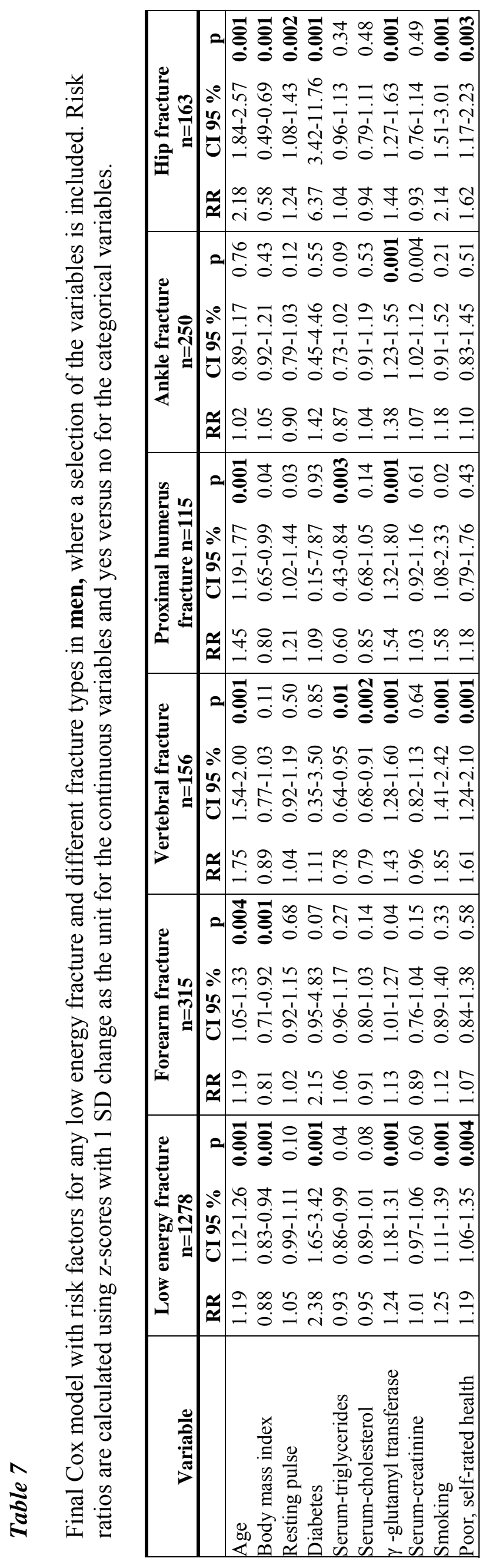





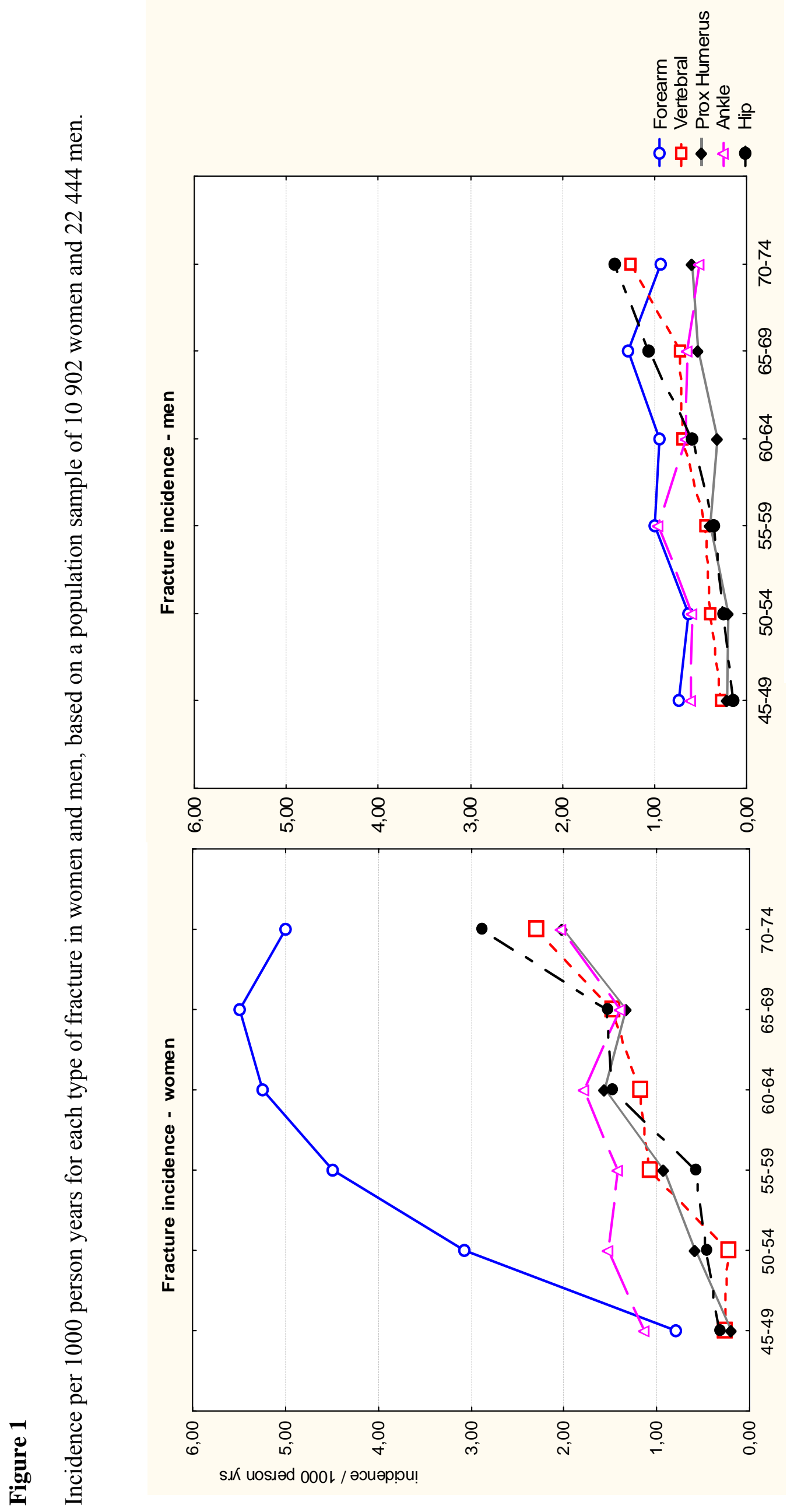





\section{References}

1. Obrant, K.J., et al., Increasing age-adjusted risk of fragility fractures: a sign of increasing osteoporosis in successive generations? Calcif Tissue Int, 1989. 44(3): p. 157-67.

2. Kannus, P., et al., Why is the age-standardized incidence of low-trauma fractures rising in many elderly populations? J Bone Miner Res, 2002. 17(8): p. 1363-7.

3. van Staa, T.P., et al., Epidemiology of fractures in England and Wales. Bone, 2001. 29(6): p. 517-22.

4. Kanis, J.A. and F.A. Pitt, Epidemiology of osteoporosis. Bone, 1992. 13 Suppl 1: p. S7-15.

5. Melton, L.J., 3rd, et al., Cost-equivalence of different osteoporotic fractures. Osteoporos Int, 2003. 14(5): p. 383-8.

6. Ettinger, B., et al., Limb fractures in elderly men as indicators of subsequent fracture risk. Arch Intern Med, 2003. 163(22): p. 2741-7.

7. Klotzbuecher, C.M., et al., Patients with prior fractures have an increased risk of future fractures: a summary of the literature and statistical synthesis. J Bone Miner Res, 2000. 15(4): p. 721-39.

8. Johnell, O., et al., Fracture risk following an osteoporotic fracture. Osteoporos Int, 2004. 15(3): p. 175-9.

9. Berglund, G., et al., Cardiovascular risk groups and mortality in an urban swedish male population: the Malmo Preventive Project. J Intern Med, 1996. 239(6): p. 48997.

10. Holmberg, A., et al., Forearm bone mineral density in 1294 middle-aged women: a strong predictor of fragility fractures. J Clin Densitom, 2004. 7(4): p. 419-23.

11. Holmberg, A.H., et al., Risk factors for hip fractures in a middle-aged population: a study of 33,000 men and women. Osteoporos Int, 2005. 16(12): p. 2185-94.

12. Jonsson, B., et al., Life-style and different fracture prevalence: a cross-sectional comparative population-based study. Calcif Tissue Int, 1993. 52(6): p. 425-33.

13. Sanders, K.M., et al., Age- and gender-specific rate of fractures in Australia: a population-based study. Osteoporos Int, 1999. 10(3): p. 240-7.

14. Kanis, J.A., et al., Long-term risk of osteoporotic fracture in Malmo. Osteoporos Int, 2000. 11(8): p. 669-74.

15. Melton, L.J., 3rd, et al., Long-term trends in the incidence of distal forearm fractures. Osteoporos Int, 1998. 8(4): p. 341-8. 
16. Chu, S.P., et al., Risk factors for proximal humerus fracture. Am J Epidemiol, 2004. 160(4): p. 360-7.

17. Nguyen, T.V., et al., Risk factors for proximal humerus, forearm, and wrist fractures in elderly men and women: the Dubbo Osteoporosis Epidemiology Study. Am J Epidemiol, 2001. 153(6): p. 587-95.

18. Olsson, C., A. Nordqvist, and C.J. Petersson, Increased fragility in patients with fracture of the proximal humerus: a case control study. Bone, 2004. 34(6): p. 1072-7.

19. Guggenbuhl, P., J. Meadeb, and G. Chales, Osteoporotic fractures of the proximal humerus, pelvis, and ankle: epidemiology and diagnosis. Joint Bone Spine, 2005. 72(5): p. 372-5.

20. Honkanen, R., et al., Relationships between risk factors and fractures differ by type of fracture: a population-based study of 12,192 perimenopausal women. Osteoporos Int, 1998. 8(1): p. 25-31.

21. Valtola, A., et al., Lifestyle and other factors predict ankle fractures in perimenopausal women: a population-based prospective cohort study. Bone, 2002. 30(1): p. 238-42.

22. Seeley, D.G., et al., Predictors of ankle and foot fractures in older women. The Study of Osteoporotic Fractures Research Group. J Bone Miner Res, 1996. 11(9): p. 134755 .

23. Ahmed, L.A., et al., Diabetes mellitus and the risk of non-vertebral fractures: the Tromso study. Osteoporos Int, 2005: p. 1-6.

24. Forsen, L., et al., Diabetes mellitus and the incidence of hip fracture: results from the Nord-Trondelag Health Survey. Diabetologia, 1999. 42(8): p. 920-5.

25. Ottenbacher, K.J., et al., Diabetes mellitus as a risk factor for hip fracture in mexican american older adults. J Gerontol A Biol Sci Med Sci, 2002. 57(10): p. M648-53.

26. Schwartz, A.V., et al., Older women with diabetes have an increased risk of fracture: a prospective study. J Clin Endocrinol Metab, 2001. 86(1): p. 32-8.

27. Vestergaard, P., L. Rejnmark, and L. Mosekilde, Relative fracture risk in patients with diabetes mellitus, and the impact of insulin and oral antidiabetic medication on relative fracture risk. Diabetologia, 2005. 48(7): p. 1292-9.

28. Adami, S., et al., Relationship between lipids and bone mass in 2 cohorts of healthy women and men. Calcif Tissue Int, 2004. 74(2): p. 136-42.

29. Lidfeldt, J., et al., The influence of hormonal status and features of the metabolic syndrome on bone density: a population-based study of Swedish women aged 50 to 59 years. The women's health in the Lund area study. Metabolism, 2002. 51(2): p. 26770.

30. Conigrave, K.M., et al., Traditional markers of excessive alcohol use. Addiction, 2003. 98 Suppl 2: p. 31-43. 
31. Conigrave, K.M., J.B. Saunders, and J.B. Whitfield, Diagnostic tests for alcohol consumption. Alcohol Alcohol, 1995. 30(1): p. 13-26.

32. Banciu, T., et al., Serum gamma-glutamyltranspeptidase assay in the detection of alcohol consumers and in the early and stadial diagnosis of alcoholic liver disease. Med Interne, 1983. 21(1): p. 23-9.

33. Trell, E., H. Kristenson, and G. Fex, Alcohol-related problems in middle-aged men with elevated serum gamma-glutamyltransferase: a preventive medical investigation. $\mathrm{J}$ Stud Alcohol, 1984. 45(4): p. 302-9.

34. Yokoyama, H., et al., Association between gamma-glutamyl transpeptidase activity and status of disorders constituting insulin resistance syndrome. Alcohol Clin Exp Res, 2003. 27(8 Suppl): p. 22S-25S.

35. Kanis, J., et al., Risk factors for hip fracture in men from southern Europe: the MEDOS study. Mediterranean Osteoporosis Study. Osteoporos Int, 1999. 9(1): p. 4554.

36. Johnell, O., et al., Risk factors for hip fracture in European women: the MEDOS Study. Mediterranean Osteoporosis Study. J Bone Miner Res, 1995. 10(11): p. 180215.

37. Clark, M.K., et al., Bone mineral density and fractures among alcohol-dependent women in treatment and in recovery. Osteoporos Int, 2003. 14(5): p. 396-403.

38. Kanis, J.A. and O. Johnell, Requirements for DXA for the management of osteoporosis in Europe. Osteoporos Int, 2005. 16(3): p. 229-38.

39. Law, M.R. and A.K. Hackshaw, A meta-analysis of cigarette smoking, bone mineral density and risk of hip fracture: recognition of a major effect. Bmj, 1997. 315(7112): p. 841-6.

40. Huopio, J., et al., Risk factors for perimenopausal fractures: a prospective study. Osteoporos Int, 2000. 11(3): p. 219-27.

41. Kiel, D.P., et al., The effect of smoking at different life stages on bone mineral density in elderly men and women. Osteoporos Int, 1996. 6(3): p. 240-8.

42. Gerdhem, P. and K.J. Obrant, Effects of cigarette-smoking on bone mass as assessed by dual-energy $X$-ray absorptiometry and ultrasound. Osteoporos Int, 2002. 13(12): p. 932-6.

43. Ahmed, L.A., et al., Self-reported diseases and the risk of non-vertebral fractures: the Tromso study. Osteoporos Int, 2006. 17(1): p. 46-53. 